\title{
La logique de l'eoikos et ses transformations : Xénophane, Parménide, Platon
}

\section{Maria Michela Sassi}

\section{(2) OpenEdition}

1 Journals

\section{Édition électronique}

URL : https://journals.openedition.org/philosant/898

DOI : 10.4000/philosant.898

ISSN : 2648-2789

Éditeur

Éditions Vrin

\section{Édition imprimée}

Date de publication : 1 novembre 2013

Pagination : 13-35

ISBN : 978-2-7574-0591-8

ISSN : 1634-4561

\section{Référence électronique}

Maria Michela Sassi, «La logique de l'eoikos et ses transformations : Xénophane, Parménide, Platon », Philosophie antique [En ligne], 13 | 2013, mis en ligne le 01 novembre 2018, consulté le 05 décembre 2022. URL : http://journals.openedition.org/philosant/898 ; DOI : https://doi.org/10.4000/philosant. 898

\section{(c) (i) ()}

Creative Commons - Attribution - Pas d'Utilisation Commerciale - Pas de Modification 4.0 International - CC BY-NC-ND 4.0

https://creativecommons.org/licenses/by-nc-nd/4.0/ 


\section{LA LOGIQUE DE L'EOIKOS ET SES TRANSFORMATIONS : XÉNOPHANE, PARMÉNIDE, PLATON* \\ Maria Michela SASSI \\ Université de Pise}

RÉSUMÉ. L'adjectif eoikos apparaît dans trois passages cruciaux de la pensée grecque (Xénophane, fr. B35; Parménide, fr. B8, 60 ; Platon, Timée, 29b3-c3), caractérisant une certaine « ressemblance » à la vérité qui se veut constitutive du discours du savant. En fait, le long de cet examen on découvre que les trois usages du terme ne peuvent pas être disposés le long d'une ligne continue, vu la difficulté de comprendre à quelle notion de vérité, sous quel point de vue, et avec quel degré d'évidence, ce discours se veut « ressemblant » selon l'occasion. Dans le cas de Parménide, cet aspect passe même au second plan, puisqu'on assiste à la prédominance d'une autre connotation qui accompagne le terme eoikos à partir de l'usage homérique, à savoir celle de la « convenance » à un contexte communicatif. L'article cherche dans l'ensemble à éclairer les déplacements que subit la logique de la similitude (et des procédés analogiques qui s'y greffent) en passant par des cadres ontologiques et épistémologiques aussi différents que le sont ceux de Xénophane, Parménide et Platon.

SUMMARY. The adjective eoikos appears in three crucial passages of Greek thought (Xenophanes, fr. B35; Parmenides, fr. B8, 60; Plato, Timaeus, 29b3-c3), in all of them expressing the notion of "resemblance" to the truth that is considered to be fundamental to the scientific discourse. In fact, this discussion shows that the three uses of the term cannot be arranged along a continuous line, owing to the difficulty in discerning what notion of truth, in what respect, and to what degree of clarity, this

:Cet article est la version fortement remaniée de la communication présentée au colloque dédié à L'analogie: un mode de connaissance en Grèce ancienne, tenu au Centre Léon Robin les 11 et 12 Février 2011. Je tiens à remercier Leopoldo Iribarren et André Laks de m'avoir invitée, et avec eux tous les participants à la discussion qui m'ont proposé des critiques et suggestions fort utiles, notamment Gabor Betegh, Pierre Judet de la Combe, Glenn Most. Je suis particulièrement reconnaissante à Leopoldo Iribarren et à Gérard Journée pour m'avoir aussi généreusement aidée à affiner l'écriture de ce texte ainsi que mes traductions des fragments des auteurs présocratiques cités.

Philosophie antique, $\mathrm{n}^{\circ} 13$ (2013), 13-35 
discourse should be "like" from time to time. In the case of Parmenides, this aspect seems even to be of secondary importance, as in the term prevails another connotation, which accompanies eoikos from Homer onwards, namely, that of "convenience" of a speech to a communicative context. The article seeks to enlighten the complicate course of the logic of similarity (and of the analogical procedures engaging with it) through such diverse ontological and epistemological frameworks as are those of Xenophanes, Parmenides, and the late Plato. 


\section{Introduction}

Au moins depuis la publication en 1966 du travail pionnier de Geoffrey Lloyd, il ne fait aucun doute que le procédé analogique doit être considéré, autant que celui fondé sur la polarité, comme un mode de connaissance constitutif de la pensée grecque, pas seulement archaïque (ni, en fait, seulement grecque). Polarity and Analogy a inspiré dans le dernier demi-siècle d'innombrables recherches et analyses consacrées aux utilisations spécifiques qui ont été faites de cet instrument cognitif, selon des modes multiples, chez plusieurs auteurs de la littérature grecque (aussi bien que latine) ${ }^{1}$.

Une place tout à fait centrale dans ce cadre est sûrement occupée par la notion de ressemblance, en tant que moteur d'un procédé de comparaison qui émerge dès Homère comme la démarche embryonnaire du raisonnement analogique, et donc comme un trait fondamental de la pensée archaïque. C'est le cas évidemment des similitudes homériques, où la comparaison agit comme un acte de connaissance, particulièrement dans les cas où un être humain est comparé à un dieu ou à un animal. En fait, la similitude homérique avait déjà reçu l'attention méritée d'autres chercheurs quelques décennies avant l'étude de Lloyd : les pages que Bruno Snell lui a dédiées dans son ouvrage désormais classique sont à cet égard incontournables ${ }^{2}$. Toutefois, en vue de l'analyse que je vais proposer, je voudrais m'arrêter plutôt sur un essai toujours stimulant d'André Rivier, Un emploi archaïque de l'analogie chez Héraclite et Thucydide (1952). Dès le début de ce petit livre, l'auteur donnait une indication qui a été pour moi, depuis longtemps, une source d'inspiration ${ }^{3}$ :

[...] cette confrontation fait paraître l'intérêt d'une enquête plus large qui,

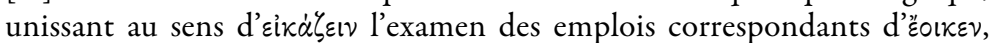

1. Il suffira ici de rappeler Schiesaro 1990.

2. Snell 1946. Voir aussi Fränkel 1921.

3. Je vais développer dans cet essai des idées que j'ai esquissées très brièvement il y a quelques années (Sassi 2005). 


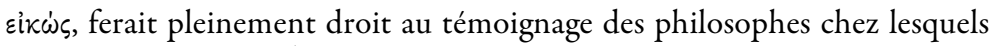
ceux-ci sont attestés (singulièrement Xénophane 21 B 34,3 s. et 35, Parménide 28 B 1,31 s. et 8,60) et dégagerait de façon générale ce sens des « ressemblances » comme une structure favorite de la pensée archaïque. Dès lors, il deviendrait tentant de se demander quel fut le sort de ces termes aux $V^{e}$ et IV siècles; et si Platon, par exemple, chez qui nous les trouvons utilisés dans de nombreux passages, comme celui du Timée (29B) où sont définis les $\lambda$ ó l'originalité de leur emploi primitif ${ }^{4}$.

Rivier appelait ainsi de ses vœux une étude des différents usages que Xénophane, Parménide et Platon font d'èoıcís, le terme par excellence de la ressemblance. Que je sache, il n'a pas poursuivi ce projet lui-même, en dehors de quelques remarques qu'on peut lire dans un important article postérieur (1956) sur les fragments B34 et B35 de Xénophane; bien que d'autres savants (qui seront cités dans la suite) aient effleuré le sujet de temps en temps, pendant longtemps personne ne l'avait choisi comme objet principal d'étude jusqu'au livre très récent de Jenny Bryan (2012). Ce livre, au titre prometteur Likeness and Likelihood in the Presocratics and Plato, est venu combler ce qui était une lacune, en mettant à juste titre en parallèle la chaîne des significations multiples dont le mot દ̇oıḱ่s se charge chez Xénophane, Parménide et Platon avec un parcours serré à travers leurs options épistémologiques et méthodologiques respectives. Néanmoins, il peut être intéressant de proposer ici une autre histoire possible, qui diffère sur certains points de la ligne dessinée par J. Bryan, bien qu'en même temps elle converge sur d'autres (les principales divergences et convergences seront dûment signalées).

En fait, disposer les usages d'żoıkc่s le long d'une ligne claire n'est pas chose aisée, ce qui s'explique à un premier niveau par la sémantique « glissante » du mot lui-même. Celui-ci a un premier sens très clair, assuré par son lien étymologique avec $\varepsilon \dot{k} \dot{\alpha} \zeta \varepsilon \iota \nu$, qui qualifie quelque chose comme «semblable » à quelque chose d'autre. Cependant une autre acception

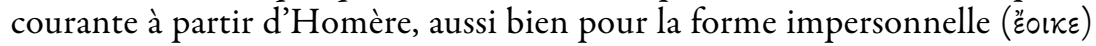

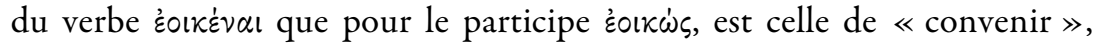
« être approprié à »; or, justement, en ce qui concerne les usages du participe dans le langage archaïque, il est souvent très difficile de déterminer quel sens prévaut dans telle ou telle occurrence, au point que, dans certains cas, nous sommes forcés d'admettre une coalescence des deux valeurs sémantiques ${ }^{5}$. Les choses se compliquent encore du fait que, dans les textes où

4. Rivier 1952, p. 8.

5. C'est un point que l'analyse toujours précieuse de Turrini 1977 a montré définitivement. Voir aussi Hoffman 2008, mettant l'accent entre autres sur le fait que le sens de «convenance » d'zixís reste important dans l'éloquence attique. Bryan 2012 exploite 
le facteur de la similitude est clair, le vecteur de celle-ci peut varier dans les directions les plus diverses selon les présupposés ontologiques et épistémologiques du contexte (à quoi, de connu ou d'inconnu, ressemble l'objet en question? sous quel point de vue? et avec quel degré d'évidence ?). De ce point de vue, on ne peut parler d'une « logique » intrinsèque à la notion de ressemblance véhiculée par le terme żoıkís (comme j'ai osé le faire dans le titre de cet essai), sinon avec la spécification qu'il s'agit d'une logique puissamment centrifuge.

La situation est plus facile à comprendre si l'on se place momentanément dans une perspective plus large, telle celle dessinée par Geoffrey Lloyd dans ses recherches axées sur la comparaison avec la science chinoise, en collaboration avec le sinologue Nathan Sivin ${ }^{6}$. Cette perspective nous invite à faire préalablement attention au fait que chez les auteurs que nous allons examiner l'analogie tend à agir sur le plan de certaines dichotomies (entre apparence et réalité, sensible et intelligible, probable et certain) qui sont tout à fait particulières au discours philosophique grec. Plus précisément, l'analogie grecque se constitue comme une pratique de médiation entre deux niveaux dont l'on présuppose la distinction, visant à déduire des facteurs causatifs inconnus à partir de leurs effets visibles, tandis que dans la pensée chinoise, où la corrélation prévaut sur la causalité, le cosmos est censé être tout ce qui existe, traversé par des correspondances que le but de l'analogie est tout simplement de mettre en évidence. Il se peut donc que l'analogie soit un mode de connaissance universel, dans la mesure où comparer et contraster sont des actes incontournables dans tout langage, bien que ses manifestations varient à la fois à travers les cultures et, pour des raisons similaires, au sein d'une même culture. C'est pourquoi je vais présenter les trois cas de l'usage d'eoikos chez Xénophane, Parménide et Platon comme autant d'actes de parole à l'intérieur de cette langue universelle qu'est l'analogie, en tant que ces actes sont déterminés par des présupposés épistémologiques et méthodologiques qui diffèrent d'un cas à l'autre. Commençons donc par une discussion du fragment 35 de Xénophane.

\section{Xénophane, B 35 DK}

Xenoph. B 35 :

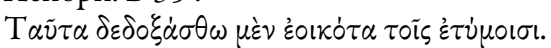

également l'ambiguité d'żoıkw่s dans sa lecture de Parménide (p. 74-78, 104-113), mais elle n’élargit cette possibilité interprétative ni à Xénophane ni au Platon du Timée, comme au contraire je vais le faire.

6. Voir, par exemple, Lloyd 1996, p. 93-139 ; Lloyd-Sivin 2002, p. 158-173. 
que ces choses soient (bien sûr?) acceptées, comme conformes aux choses réelles.

Ce vers aussi célèbre que bref nous est parvenu, malheureusement, privé de tout contexte, et nous ne savons même pas si un $\delta \dot{\varepsilon}$ adversatif pouvait figurer dans les lignes suivantes (nous assumons toutefois, dans notre lecture, que $\mu \dot{\varepsilon} \nu$ peut avoir un sens confirmatif qui n'exige aucune corrélation avec un $\delta \dot{\varepsilon}$ qui suive $)^{7}$. En revanche, nous avons là un concentré de termes à haute densité sémantique, qu'on peut essayer de déchiffrer, en toute confiance ${ }^{8}$.

Pour commencer, il est inévitable de saisir dans l'expression દ̇oıkó $\tau \alpha$

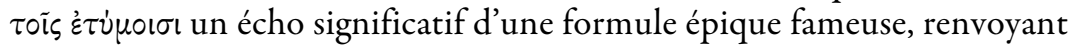
à cette capacité de forger des discours faux mais « semblables à la vérité »,

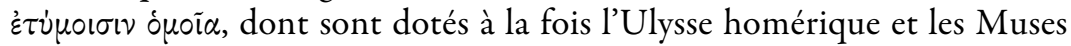
d'Hésiode.

Hom. Od. XIX 203 :

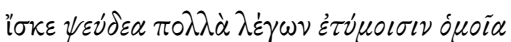

Il feignait plusieurs faussetés en disant des choses semblables à des réalités?

Hes. Theog. 26-28:

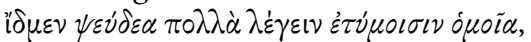

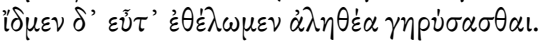

Nous savons dire plusieurs faussetés semblables à des réalités,

mais nous savons, quand nous le voulons, dire des choses vraies.

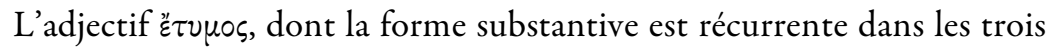

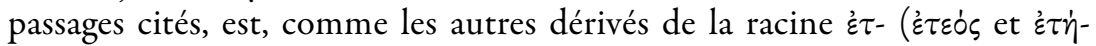

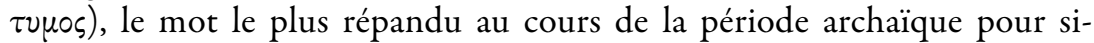
gnifier la vérité, avec une connotation objective très marquée (partagée d'ailleurs par le mot $\dot{\alpha} \lambda \eta \theta \dot{\eta} s$, qui s'installera définitivement au IV siècle) insistant sur la réalité « authentique » des choses. Dans ce contexte, ce ne peut être un hasard si Xénophane substitue દ̇oıḱ่s à ónoĩos en combinaison

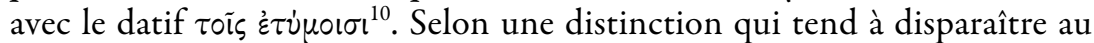
$\mathrm{V}^{\mathrm{e}}$ siècle, ónoíos peut signifier « ressemblant» aussi bien que «iden-

7. Cf. Rivier 1956, p. 354. J'ai déjà soutenu ailleurs avec d'autres arguments (Sassi 2011a) une interprétation des idées de Xénophane sur la connaissance que je déclare franchement empiriste, m'inspirant, comme déjà Rivier, de Fränkel 1925. Bryan 2012, dont l'interprétation de Xénophane s'inscrit dans la veine du «faillibilisme » (p. 6-57), préfère considérer $\mu$ '́ $\nu$ comme l'indice d'une antithèse entre l'incertitude déclarée de ses doctrines et la certitude du savoir divin, qui aurait pu apparaître dans la ligne suivante.

8. En ce sens Lesher 1992 est une aide précieuse, à ajouter aux ouvrages cités ci-dessus.

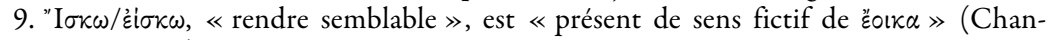
traine 1990, p. 354).

10. Voir dans le même sens Bryan 2012, p. 28-36. 
tique », et se prête à indiquer plutôt une ressemblance trompeuse au point de faire croire à une identité, tandis que દ̇oıkw่s implique une similarité reconnaissable en tant que telle par rapport à un point de référence donné, telle celle d'un fils à sa mère (Hes. $O p$. 235). S'inscrivant pleinement dans le contexte archaïque, où dire la vérité, c'est reproduire fidèlement et exac-

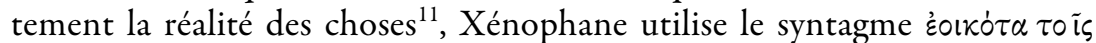

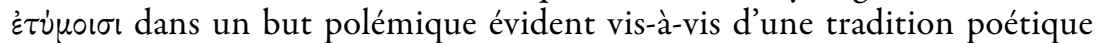
simulatrice. En s'opposant à la ressemblance trompeuse de ces discours faux qui, grâce à une construction formelle habile, peuvent se faire passer pour vérité, il vise à une représentation honnête aussi bien que conjecturale de la réalité des choses ${ }^{12}$.

L'aspiration de Xénophane à un discours autant que possible correct (autrement dit, un discours «vraisemblable ») est confirmée par l'impératif $\delta \varepsilon \delta \circ \xi \dot{\alpha} \sigma \theta \omega$, exprimant une invitation forte à formuler sur les choses des « opinions ». On pense au cadre épistémologique dessiné dans le fragment 34 , où le dókos désigne un mode de connaissance valable, en tout cas le seul qui soit concédé à l'homme, puisque la divinité seule détient cet accès direct à la réalité des choses qui aboutit à une connaissance certaine, à savoir celle des dieux et de la totalité du monde, dont Xénophane va parler cependant dans son traité. Ici, tout comme dans le fragment 1 d'Alcméon ${ }^{13}$, l'opposition de la connaissance parfaite des dieux au caractère conjectural de la connaissance humaine a l'allure d'un incipit programmatique (et je serais portée à admettre que le fragment 35 occupait également une position introductive $)^{14}$.

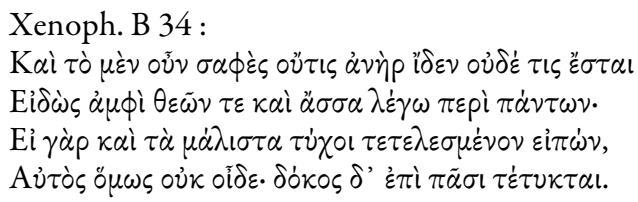

Xenoph. B 34 :

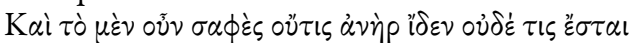

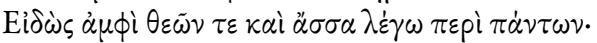

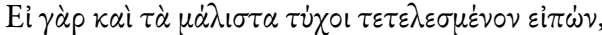

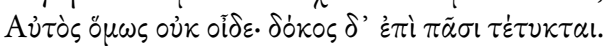

11. Cf. Cole 1983.

12. Dans les vers cités d'Homère et d'Hésiode, la puissance créatrice de la parole est soulignée respectivement par ไ̋ $\sigma \kappa \varepsilon . . . \lambda \dot{\varepsilon} \gamma \omega \nu / \lambda \dot{\varepsilon} \gamma \varepsilon \iota \nu$, et $\gamma \eta p \dot{\sigma} \sigma \alpha \sigma \theta \alpha$. Et Théognis, I, 713, de reprendre ces passages célèbres pour évoquer l'efficace créatrice d'une langue experte... telle

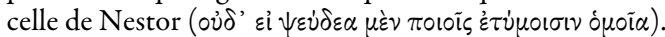

13. Je me suis arrêtée ailleurs (Sassi 2011, p. 210-213) sur le caractère programmatique des deux fragments d'Alcméon et de Xénophane, en les lisant (avec le fragment 1a d'Hécatée) comme des manifestations efficaces d'une recherche de garanties de vérité, loin de l'appel à une inspiration divine quelconque, selon la méthode particulière à la connaissance humaine.

14. Rivier 1975 , p. 359 , remarque en passant que le fragment B35 est peut-être l'épilogue de l'écrit dont B34 est le préambule. Bryan 2011, p. 8, admet les deux possibilités. 
Et bien sûr, la claire certitude, aucun homme ne l'a vue, et il n'existera pas non plus quelqu'un ayant la connaissance directe des dieux et de ce que je dis à propos de toutes choses.

Car même si quelqu'un réussissait à dire quelque chose d'accompli, toutefois il ne le saurait pas lui-même; car sur toutes les choses il y a une opinion bien construite.

Je propose pour ce dernier vers une interprétation particulière de $\tau \dot{\varepsilon} \tau v-$ $\kappa \tau \alpha \mathrm{l}$, dans la mesure où cette forme passive du parfait de $\tau \varepsilon \dot{\gamma} \chi \omega$ (« construire $\gg)$ me semble renvoyer à un aspect de construction positive d'une opinion sur « toutes les choses » (je préfère voir en $\dot{z} \pi \grave{i} \pi \tilde{\alpha} \sigma l$ une référence à l'extension des objets de l'opinion plutôt qu'au fait qu'elle soit répandue « chez tous les hommes », ce qui serait banal). Mais comment s'opère finalement, pour Xénophane, la construction d'un discours correspondant au réel ? Ou, en d'autre termes, quels sont les critères qui orientent le vecteur de la similarité recherchée ? Nous ne pouvons nous appuyer sur aucune affirmation explicite de Xénophane à ce sujet, mais on a l'impression qu'il adapte sa méthode selon les différents objets de son enquête. Pour ce qui est des événements naturels, il ne cherche pas un principe unifiant à la manière des Milésiens, mais il se borne plutôt à décrire avec précision le cycle des transformations des grandes masses cosmiques et leurs effets visibles (B 30, 31, 32). Par conséquent, son emploi du procédé par inférence est très prudent et réglé ${ }^{15}$. On peut aussi supposer que son hypothèse que la terre s'étend infiniment au-dessous de la surface que nous voyons sous nos pieds (B 28), pour hardie qu'elle puisse paraître, se veut une hypothèse «économique », en réponse aux variations plus ou moins imaginatives que Thalès, Anaximandre et Anaximène avaient proposées pour expliquer la stabilité de la terre au centre de l'univers. En définitive, ce qu'on appelle depuis Hermann Fränkel l'«empirisme »de Xénophane pourrait être reformulé, selon une heureuse définition d'Edward Hussey, comme un procédé d' «extrapolation parcimonieuse », axé sur l'idée qu'il n'y a d'autres choses ou forces agissant dans le monde que celles dont nous pouvons avoir l'expérience ${ }^{16}$. Comme Rivier l'écrivait déjà, «Xénophane n’a pas édifié de cosmologie. Il décrit l'univers » ${ }^{17}$.

15. Voir l'extrapolation du rôle cosmique de la terre et de l'eau à l'explication des phénomènes du vivant, B27, B29, B33; ou le renvoi des trouvailles de fossiles en Sicile à une période géologique passée, A13.

16. « Parsimonious extrapolation », Hussey 1990, p. 26. Hussey 2006, p. 13, va jusqu’à affirmer que Xénophane, pour être le premier auteur appliquant à sa propre recherche positive une réflexion explicite sur les conditions de la connaissance, mérite le titre de « premier philosophe ».

17. Rivier 1956, p. 365. 
Il est notable que Xénophane aborde le problème de la nature du dieu, qui occupe pour lui un espace inaccessible à l'expérience humaine, par une voie nécessairement différente mais tout à fait comparable, puisqu'il y emploie la même «parcimonie » dont il fait preuve dans l'explication des faits physiques. De fait, il lui suffit d'éradiquer toute représentation de la nature divine qui puisse être taxée de ressemblance avec les attributs humains, pour faire renaître des ruines d'un anthropomorphisme ridiculisé une figure de dieu suprême qui est construite par la négation des qualités humaines (B 23-25) ${ }^{18}$. Cette construction se fonde sur un procédé analogique inversé, assez semblable a celui qu'Hécatée de Milet met en ouvre dans sa réduction rationaliste des «contes ridicules » de la tradition mythique, selon le programme qu'il annonce au début de son écrit.

Hecat., fr. 1a Jacoby:

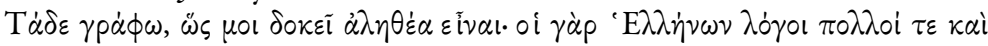

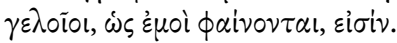

J'écris les choses qui suivent comme je les tiens pour vraies : en effet, les récits des Grecs m’apparaissent aussi nombreux que ridicules.

On retrouve ici dans une tournure très significative le verbe $\delta \circ \kappa \varepsilon \dot{\varepsilon} \omega$, qui définit un processus d'élaboration d'un point de vue critique passant (a) par une comparaison des mythes grecs entre eux, visant à mettre au jour les contradictions internes au corpus mythique; (b) par une comparaison de tel ou tel mythe avec un principe de réalité historique, consistant à juger à l'aune de l'expérience habituelle ce qui « pourrait s'être effectivement passé » dans un temps dont on ne peut avoir de connaissance directe, et à dévoiler les exagérations ou embellissements éventuels des récits traditionnels en en mesurant la crédibilité. En bref, c'est un principe d'analogie (avec le présent) qui oriente la recherche d'Hécatée, qui aboutit par exemple à nier qu'Égyptos ait pu avoir cinquante fils (tout au plus une vingtaine, dit le fr. 19 Jacoby) $)^{19}$. On peut bien dire que c'est

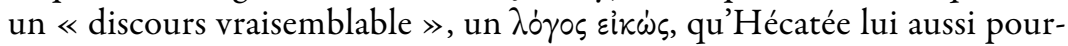
suit au moyen de l'analogie. Qu'il ait employé ou non cette expression, il est notable qu'on la trouve beaucoup plus tard chez Pausanias, qui dit

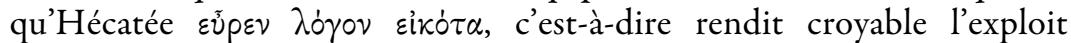
d'Héraclès avec Cerbère, en affirmant que le «chien de l'Hadès » que le

18. C'était déjà la lecture de Babut 1974 (voir en particulier p. 406-409), que j’ai développée dans un essai concentré sur la critique par Xénophane de la représentation anthropomorphique des dieux (à paraître).

19. Voir Corcella 1984, p. 48-54. 
héros captura pour Eurysthée devait être simplement un serpent à la morsure mortelle (Paus. Per. III 25, 4 = Hec. Fr. 27 Jacoby).

En tout cas ni Hécatée, ni Xénophane ne se bornent à une construction par la négative : au contraire, tous les deux s'inspirent d'une certaine idée de cohérence argumentative, capable de satisfaire les attentes d'un public qu'on peut imaginer cultivé et curieux d'un savoir religieux et mythique plus sophistiqué que d'ordinaire. De plus, Xénophane charge explicitement ce programme de l'exigence d'une vision moralisée qui soit « convenable » et « appropriée » au statut spécial de la personne divine. Cela ressort de façon évidente dans le fragment 26, où Werner Jaeger a vu percer un thème devenu par la suite central dans la pensée religieuse grecque, à savoir celui du theoprepes ${ }^{20}$. Même si on décline ce thème sur le plan éthique plutôt que théologique, comme l'a suggéré Daniel Babut ${ }^{21}$, il est évident que la critique de Xénophane dans ce fragment est fondée sur un critère de «convenance », selon lequel les allées et venues des dieux homériques ne conviennent pas à la dignité de la nature divine.

Xenoph. B 25:

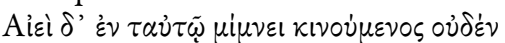

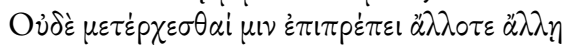

Il reste toujours dans le même lieu, ne bougeant pas du tout et il ne lui convient pas de se déplacer çà et là.

Nous dirons en somme que dans les deux champs principaux de son exploration, par delà les différentes méthodes adoptées, Xénophane se tient à une attitude « minimaliste » : ayant comme modeste but de formuler un discours qui fournit au monde un sens ordonné, il n'éprouve le besoin d'imaginer rien ou presque au-delà des phénomènes observables, dont il fait ressortir les ressemblances pour retrouver des principes unificateurs (par exemple la terre et l'eau, avec leurs transformations). Pour ce qui concerne le discours sur le divin, faute de points de repère empiriques, il recourt comme on vient de le voir à deux ressources supplémentaires (d'un côté la simple négation des caractéristiques humaines, de l'autre un critère de bienséance morale), qui néanmoins se fondent toutes deux sur l'inversion absolue du mécanisme de la similitude homérique. Dire, comme le fait habituellement Homère, qu'un individu est «semblable » au dieu par sa beauté et sa puissance physique, ou même par sa noblesse, équivaut à rabaisser le niveau du divin à l'humain. Il faut au contraire chercher une descrip- 
tion du dieu qui lui « ressemble» en tant que « convenable»: ce qu'on dirait en grec, en tout cas, un discours żokx $\omega^{22}$.

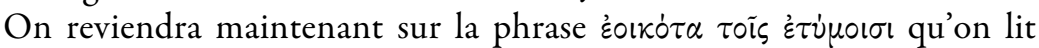
dans le fragment B35, pour remarquer finalement que l'emploi de l'adjectif żouḱs joue ici probablement à la fois sur les deux acceptions («semblable » et «convenable ») qui partagent son champ sémantique dès les poèmes homériques, et dont la deuxième garde un lien avec la notion de similitude puisqu'on désigne comme « convenable » ce qui satisfait certaines attentes rationnelles et sociales (c'est-à-dire ce qui « ressemble » à un contexte typique et s'y encadre bien). C'est pourquoi il est souvent très difficile de distinguer le sens qui prévaut dans tel ou tel emploi de l'adjectif

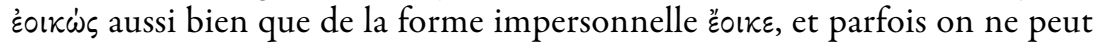
pas exclure que les deux significations soient tout simplement entremêlées dans la même occurrence. Par exemple, lorsque Dolon affirme que porter

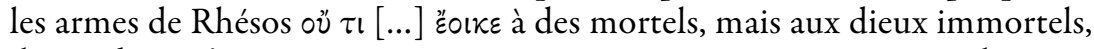
il veut dire qu'à ses yeux ces armes ne conviennent pas à un guerrier humain parce qu'elles ne ressemblent pas à son armement typique (Hom. Il. X 440). Ou encore, il n'est pas clair si les jasements d'un vieillard $\lambda$ ó $\gamma \circ 0 \varsigma \lambda \dot{\varepsilon} \gamma \omega \nu$

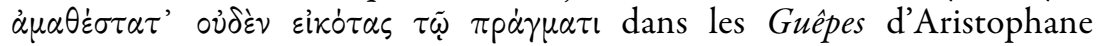
(1321) sont ainsi qualifiés parce qu'ils manquent d'opportunité ou plutôt parce qu'ils reflètent une perception erronée de la réalité qui l'entoure : les deux lectures sont possibles simultanément, car elles répondent aux nuances diverses d'une même situation ${ }^{23}$. C'est justement ce qui se passe peut-être avec les opinions que Xénophane formule dans son écrit, avec l'intention qu'elles soient « semblables » autant que « convenables» à la vérité de la nature et du divin. En tout cas, ces opinions ne sont pas « probables » dans le sens que le langage scientifique moderne donne à ce

22. Voir ci-dessus, p. 16. Il est remarquable que le lien entre ressemblance et convenance se soit conservé dans plusieurs usages linguistiques modernes : par exemple, d'un individu se conduisant en dessous de ses standards moraux habituels, on peut dire en français « cela ne lui ressemble pas », en italien «non gli somiglia », en anglais «it is not like him to do that », en allemand «das sieht nicht ihm gleich ». Rappelons également la dérivation de l'adjectif anglais seemly (décent) du verbe seem.

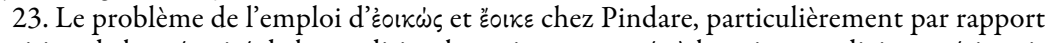
à sa vision de la précarité de la condition humaine comparée à la puissance divine, mériterait une analyse spécifique. Je me borne ici à rassembler quelques passages de son œuvre selon la traduction qui me paraît la plus probable (en tant que dominante plutôt qu'exclusive). «Convenance, justesse » : Ol. I 35 (voir Gerber 1982, p. 70-71 et Verdenius 1988, p. 22, rappelant tous les deux le fr. 26 de Xénophane), IV 27 ; Pyth. II 29, III 59 ; Nem. II 10, VII 58 ; Isthm. I 52; fr. 42.7. «Vraisemblance : Pyth. I 34, II 29. «Convenance cum vraisemblance » : Ol. XIII 102 (voir Boeke 2007, p. 135-136); Nem. III 20, IV 32 ; Isthm. V 24. 
mot: dans un monde pré-statistique ${ }^{24}$, il n'est pas du tout question d'un accroissement de données empiriques de nature à transformer des connaissances probables en des connaissances certaines. Par ailleurs, pour Xénophane tout comme pour Alcméon, ce n'est pas à la certitude parfaite sur les choses qui échappent aux sens que l'intelligence humaine peut et doit aspirer, son but propre étant une connaissance probable dans le sens du latin probabilis, c'est-à-dire une connaissance qui mérite d'être appréciée pour son contenu, si limité qu'il puisse être, et reçue.

\section{Parménide, B8.60}

Dans les dernières lignes du fragment 8 de Parménide la déesse annonce que, après avoir conclu son logos tout à fait « fiable » $(\pi เ \sigma \tau \dot{o} v)$ sur la vérité, elle va en prononcer un autre sur les opinions mortelles. Celui-ci sera le contenu de la Doxa, comme on nomme la seconde partie du poème : nous avons donc ici une sorte de deuxième prologue, dans lequel se concentrent nombre de mots significatifs du programme philosophique de Parménide, au premier rang desquels notre adjectif żoık'่s, au vers 60, dont l'interprétation est très discutée.

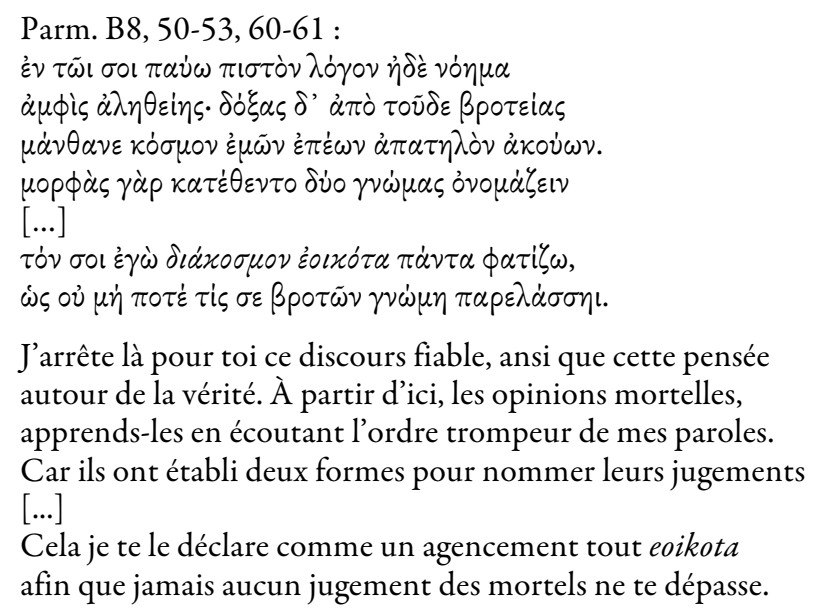

J'arrête là pour toi ce discours fiable, ansi que cette pensée autour de la vérité. À partir d'ici, les opinions mortelles, apprends-les en écoutant l'ordre trompeur de mes paroles.

Car ils ont établi deux formes pour nommer leurs jugements [...]

Cela je te le déclare comme un agencement tout eoikota

afin que jamais aucun jugement des mortels ne te dépasse.

Le lecteur du poème, arrivé au bout de l'exposition de l'Aletheia, ne s'étonne pas trop du fait que la déesse met en garde le savant en face du monde du devenir sensible, dont elle est en train d'entamer la description trompeuse. Comme on pouvait s'y attendre, cet exposé va être aussi bien organisé dans sa construction formelle (telle la connotation esthétique de

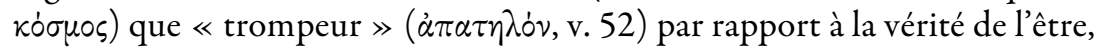


si éloignée de l'apparence changeante des phénomènes. La force de l'adjectif $\alpha \dot{\alpha} \alpha \tau \eta \lambda o^{\prime} v$ ne peut être sous-estimée, comme le montre entre autres la reprise qu'Empédocle, B17.26, fera du vers de Parménide dans une variation efficace sur le thème de l'illusion sensible comme tromperie : $\sigma \dot{\nu} \delta$ ' äkove

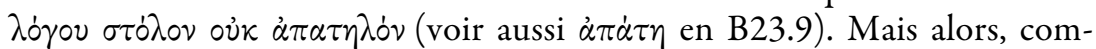
ment ne pas s'étonner en découvrant, quelques vers plus loin, que la déesse promet tout de même une description żokxó $\tau \alpha$ (v. 61) ? Si c'est «semblable », à quoi ? et si c'est « convenable », à quoi encore une fois ?

En fait, la déesse va s'arrêter longtemps sur le monde de la doxa, comme l'indique le nombre de fragments et témoignages qu'on peut attribuer à cette partie du poème de Parménide. Au vers 60 du fr. 8 , elle promet déjà un traitement exhaustif $(\pi \dot{\alpha} \nu \tau \alpha)$ de ce $\delta i \dot{\alpha} \kappa o \sigma \mu o s$ qui fait l'objet de son deuxième logos. Le choix du mot $\delta i \dot{\alpha} k o \sigma \mu o s$ nous assure, par le $\delta i \dot{\alpha}$ qui entre dans sa composition, qu'il s'agit là d'un système cosmologique complexe, qui exige qu'on lui prête une attention prolongée. Et nous savons qu'il s'agit d'un système qui développe de façon articulée la spéculation milésienne, se fondant sur l'identification de deux formes ( $\mu \rho p \phi \alpha i$ ) ou principes fondamentaux, la lumière et l'obscurité ou bien le jour et la nuit, qui pénètrent tout (fr. B9), et qu'y sont incluses également, entre autres, une théorie physique de la connaissance et des notions d'embryologie et de médecine ${ }^{25}$.

Rappelons que déjà à la fin du proème, en dévoilant finalement au kouros le but de son voyage, la déesse avait anticipé que le contenu de sa révélation serait double (une annonce qui rappelle l'ambivalence des Muses d'Hésiode à l'égard de la vérité). En effet, obligation lui serait faite d'apprendre un « tout » incluant non seulement l'a $\lambda \dot{\eta} \theta \varepsilon ı$, mais aussi les $\delta o^{\prime} \xi \alpha \iota$ des mortels : bien que dépourvues de $\pi i \sigma \tau \iota \varsigma \dot{\alpha} \lambda \eta \theta \dot{\eta} \varsigma$, du fait que tout ce que

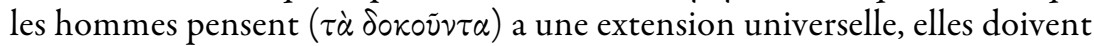

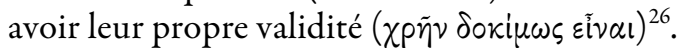

Parm. B1, 28-32:

[...] $\chi \rho \varepsilon \dot{\omega} \delta \dot{\varepsilon} \sigma \varepsilon \pi \dot{\alpha} \nu \tau \alpha \pi \nu \theta \dot{\varepsilon} \sigma \theta \alpha \iota$

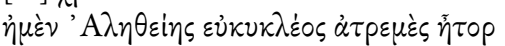

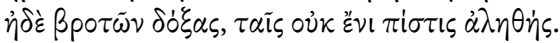

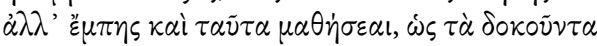

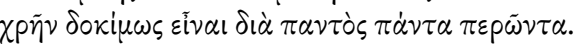

[...] il faut que tu sois instruit de tout,

25. Je reste convaincue de la distribution des textes acceptée dans les Vorsokratiker de Diels et Kranz, contra Cordero 2010, qui propose une réduction drastique de l'extension réservée à la doxa dans le poème. J'adhère évidemment à la réhabilitation du contenu de la doxa qui s'est affirmée dernièrement (cf. p. ex. Curd 1998).

26. En m'appuyant sur la lecture de Verdenius 1942, p. $49-50$ et de Mansfeld-Primavesi

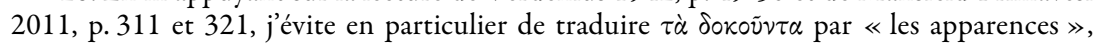
comme le font plusieurs commentateurs. 
d'une part du cœur sans tremblement de la vérité bien ronde, et, d'autre part, des opinions des mortels où il n'est aucune certitude vraie. Mais tu apprendras en plus ceci : que les choses établies

il fallait qu'elles soient validement, traversant le tout d'un bout à l'autre ${ }^{27}$.

Si nous admettons, d'après cette lecture, que la doxa a sa propre validité, bien que dans un milieu cognitif complètement séparé de celui de l'aletheia, nous pouvons peut-être comprendre pourquoi la déesse y consacre autant d'énergie. Son discours, bien qu'illusoire pour la plupart des hommes, qui prennent la réalité du monde physique pour « tout ce qui existe », peut ne pas être absolument faux (le mot serait $\psi \varepsilon v \delta \eta_{\text {s }}$ ) pour le savant qui, ayant été instruit par la déesse sur les attributs de l'être, est devenu capable de distinguer le niveau du sensible de celui de l'être. Parménide ne risque pas de rester emprisonné dans les apparences sensibles, précisément parce que la déesse joue cartes sur table : elle le préviendra, au moment opportun, face à la déception intrinsèque au monde de la doxa (B 8.52).

Cette lecture permet également d'établir un lien significatif, analogue à celui qu'on a constaté dans le cas de Xénophane, entre le champ de l'opinion (clairement défini par la répétition en trois vers consécutifs de trois

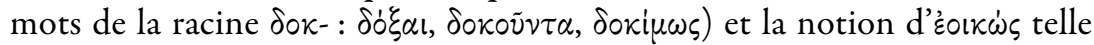
que nous la trouvons dans B $8.60^{28}$. Il paraît probable, à la lumière de la comparaison avec la fin du fragment 1 , que le mot żoเkó $\tau \alpha$ qualifie le contenu de la cosmologie comme ayant une valeur positive, donc comme « plausible ». Mais cette propriété ne semble pas dériver premièrement du fait que le discours qui suit ressemble à un modèle tel que la représentation de l'être, vu que żoukís n'est pas attesté avec le sens de « semblable » avant Platon, là où il manque un objet au datif auquel référer le rapport de similitude ${ }^{29}$. Or chez Homère le mot żoık signifie « convenable » et caractérise, au moins dans deux passages très significatifs, un discours qui se veut «approprié » aux attentes de l'auditoire, puisque correspondant non pas tant à la vérité des choses qu'à la nature du contexte communicatif donné : en d'autres termes, un discours qui se fait apprécier non seulement pour son adéquation à la vérité, mais aussi pour sa valeur autonome de constructeur de sens. Cela vaut pour les $\mu \tilde{\nu} \theta_{\circ}$

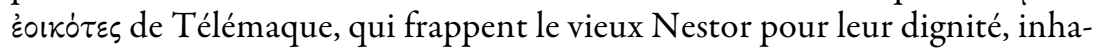

27. Je reprends ici la traduction efficace de dì̀ $\pi \alpha \nu \tau \dot{s}$ s proposée par Année 2012, p. 157.

28. Mourelatos 2008, p. 194-205, analyse le sens d' « acceptation » qui ressort de l'emploi de ces mots, caractérisant la doxa de Parménide comme une opinon reçue, c'est-à-dire normalement « acceptée ».

29. Voir Turrini 1977, en particulier p. 551 et 556. En tout cas, il n'est plus d'usage d'entendre $\delta i \dot{\alpha} \kappa \circ \sigma \mu \circ \zeta$ żoıkó $\tau \alpha$ comme un « agencement (seulement) apparent », donc franchement faux, comme le faisaient autrefois certains interprètes (cités par Verdenius 1942, p. 50 et n. 5$)$. 
bituelle pour un si jeune homme (Od. III 124-125), et vaut surtout pour la façon dont Hélène introduit sa narration des exploits d'Ulysse, se déclarant

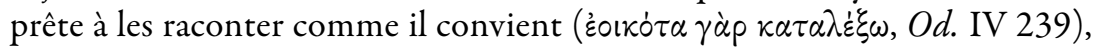
c'est-à-dire par une construction du discours articulée qui réjouisse les convives curieux en leur donnant, sinon tous les détails de l'histoire, du moins une idée de la valeur du héros (et Ménélas d'observer ensuite, v. $266: \tau \alpha \tilde{\tau} \tau \alpha$

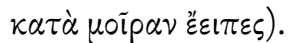

Ces parallèles peuvent confirmer que le contenu de la doxa est « plausible » non pas (ou non seulement) en tant que « semblable» en quelque chose à l'Être, mais surtout en tant qu' « appropriée » au programme d'instruction de la déesse. C'est pourquoi celle-ci livre à Parménide une cosmologie qui veut être sophistiquée, la meilleure possible peut-être, qu'aucune autre doctrine des autres mortels ne pourra dépasser ( $\dot{\omega} \varsigma$ ovj $\mu \dot{\eta} \pi \circ \tau \dot{\varepsilon} \tau \dot{\tau}$, $\sigma \varepsilon \beta \rho \circ \tau \tilde{\omega} \nu \gamma \nu \dot{\omega} \mu \eta \pi \alpha \rho \varepsilon \lambda \dot{\alpha} \sigma \sigma \eta \iota)$.

L'usage que Parménide fait du terme żokx's est donc très différent de

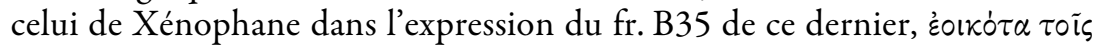

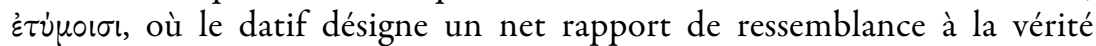
des/sur les choses ${ }^{30}$. D'autre part, comme nous l'avons vu, Xénophane vise à construire un réseau de liaisons illuminant tout à l'intérieur du monde de l'expérience, tandis qu'il serait difficile d'attribuer à Parménide la thèse qu'une représentation du monde sensible soit, sans nuances, « semblable » à la vérité de l'être intelligible ${ }^{31}$, même si l'on admet que le mécanisme analogique puisse être à l'œuvre à d'autres niveaux, par exemple dans le rapport entre la doxa, ou se mêlent la Lumière et la Nuit, et l'itinéraire de Parménide le long du chemin ou s'alternent le Jour et la Nuit ${ }^{32}$; ou bien encore entre les procédés de construction de la cosmologie et de l'étant, dans la mesure où tous deux se fondent sur l'application de certaines marques distinctives $(\sigma \dot{\eta} \mu \alpha \tau \alpha)$, d'une part à l'Être (B 8.2), de l'autre au Feu et à la Nuit (B $8.55)^{33}$.

La variation du sens d'żoık'่ qu'on peut constater chez les deux auteurs est donc l'indice d'une divergence nette sur le plan ontologique et épistémologique. Xénophane réfléchissait sur l'existence de certaines réalités qui

30. Bryan 2012, p. 64, suppose que l'absence du datif chez Parménide reflète une intention polémique à l'égard du faillibilisme de Xénophane, dans le cadre d'une épistémologie optimiste selon laquelle il est tout à fait possible aux hommes d'atteindre la connaissance de la totalité du réel (voir p. 100-113). Tout en admettant que le recours à la révélation, et en particulier l'intégration à celle-ci de la doxa, constitue un fondement stable du savoir qui donne une impulsion positive à l'enquête de Parménide, je pense que la position de Xénophane sur la connaissance est néanmoins positive à sa manière.

31. Voir Gerson 2009, p. 18 n. 4.

32. C'est la thèse de Primavesi 2005, en particulier p. 82.

33. Je dois d'importantes précisions sur ce point à un essai inédit de Leopoldo Iribarren sur « La sphère de Parménide (B 8.42-49) : L'analogie comme problème ». 
se soustraient à la connaissance sensible, mais ces réalités font partie, à ses yeux, de la physis (y compris le divin), et elles peuvent faire l'objet d'une opinion «vraisemblable » dans la mesure où la vérité filtre, pour ainsi dire, entre les mailles du sensible. Parménide revendique au contraire la possibilité d'atteindre la vérité directement, mais à condition de la poser dans un domaine intelligible, tout à fait séparé de celui de la doxa, qui vient à coïncider pour son compte avec l'apparence du sensible (c'est ici, en fait, que naît le concept d'apparence). Toutefois, du moins d'après les textes qui nous sont parvenus, Parménide ne précise pas la relation entre le sensible et l'intelligible, bien qu'il l'implique, et son discours sur le cosmos semble se modeler sur l'exigence de lui restituer un ordre quelconque plutôt que sur un raisonnement par ressemblance avec les attributs de l'être (qui est seulement effleuré dans B8.55 sqq.). On va voir maintenant que l'emploi par Platon du mot દ̇oık ultérieure de penser la ressemblance dans un cadre ontologique et épistémologique nouveau, et cependant construit sur les fondations jetées par Xénophane et Parménide.

\section{Platon, Timée, 29b3-c3}

Qu'il suffise d'évoquer ici l'image de la ligne dans la République pour rappeler que Platon développe la distinction entre le sensible et l'intelligible déjà posée par Parménide dans un cadre analogique complexe, et cependant clairement ordonné selon une hiérarchie de degrés de réalité auxquels correspondent autant de facultés cognitives ${ }^{34}$. Les quatre segments de la ligne sont réduits à deux dans le dialogue cosmologique ${ }^{35}$, précisément dans le passage introductif où Timée déclare qu'il va livrer un discours eikús (à noter que nous sommes encore dans un prologue, comme c'était le cas pour les déclarations programmatiques de Xénophane et de Parménide... et de l'Hélène d'Homère). L'affirmation selon laquelle l'être intelligible est au devenir sensible ce que la vérité est à la croyance signale une analogie indubitable (voire une égalité de rapports : l'expression $\alpha \nu \alpha ̀ \lambda$ óyov a ici une valeur technique) entre les deux niveaux ontologiques du sensible et de l'intelligible, d'un côté, et les modes de connaissance correspondants, de l'autre. La règle de la proportion fait en sorte que l'analogie fonctionne également entre les rapports que les $\lambda$ ó $\gamma o$ en entretiennent avec leurs objets : le discours qui porte sur le sensible, ou mieux sur le mode de sa génération par une

34. La littérature sur ce sujet étant considérable, je me borne à citer l'étude très précise et balancée de Franco Repellini 2003.

35. Sur les problèmes posés par cette opération, voir Grasso 2012. 
figure divine, qui échappe à la vue directe ${ }^{36}$, ne peut être qu'un mythos dépourvu de l'exactitude et de la stabilité que seul peut avoir un discours qui porte sur l'intelligible. Ce discours sera donc eikw่s puisque « apparenté » ( $\sigma \gamma \gamma \varepsilon \nu \eta ่ \varsigma)$ au devenir, qui est une image ( $\varepsilon \dot{\imath} \kappa \omega \dot{\omega}$ ) de l'être intelligible qui est son modèle $(\pi \alpha \rho \dot{\alpha} \delta \varepsilon \iota \gamma \mu \alpha)$ : mais le statut d'image du cosmos est chargé dans le Timée d'une nouvelle valeur positive ${ }^{37}$, qui se transmet, par un jeu im-

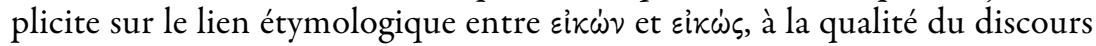
qui le concerne.

Plat. Tim. 29b3-d3 :

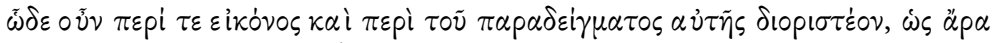

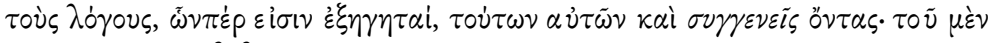

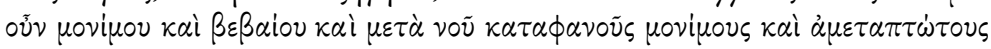

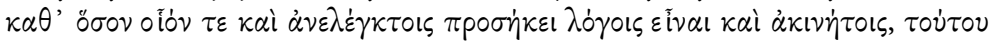

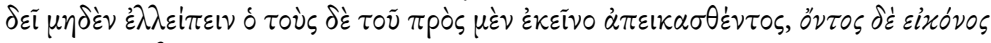

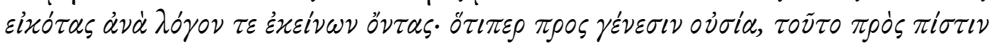

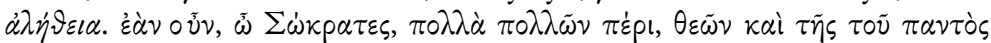

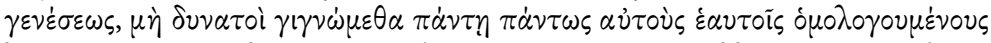

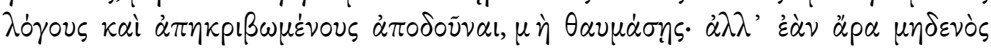

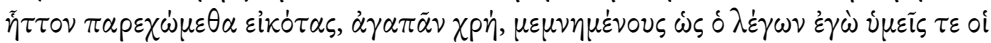

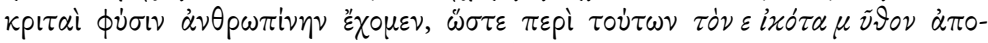

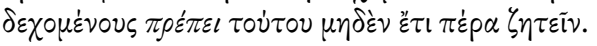

Voici donc, concernant une image et son modèle, la distinction qu'il faut établir, étant admis que tout discours porte sur quelque chose et que ce sur quoi porte ce discours lui est apparenté. D'un côté donc, tout discours qui porte sur ce qui demeure, sur ce qui est stable et translucide pour l'intellect, cela ne doit en rien manquer d'être stable et inébranlable, pour autant qu'il est possible et qu'il convient à un discours d'être irréfutable et invincible ; d'un autre côté, tout discours qui porte sur ce qui est la copie de ce dont on vient de parler, parce qu'il s'agit d'une copie, entretient avec la première espèce de discours un rapport d'image à modèle. Ce que l'être est au devenir, la vérité l'est à la croyance. Si donc, Socrate, en bien des points et sur bien des questions - les dieux et la génération de l'univers -, nous nous trouvons dans l'impossibilité de proposer des explications qui en tous points soient totalement cohérentes avec elles-mêmes et parfaitement exactes, n'en sois pas étonné. Mais, si nous proposons des explications qui ne sont pas des

36. C'est une des marques du champ mythique, comme l'a aussi rappelé récemment Brisson 2012, justement à propos du Timée.

37. Sur le statut de $\varepsilon i \kappa \omega \dot{v} / \varepsilon i k \omega \dot{s}$ dans l'œuvre plus tardive de Platon (en particulier dans le Cratyle et le Sophiste), voir Turrini 1979. Pour ce qui concerne spécifiquement le Timée, voir Bryan 2012, p. 118-130, 160, dont je partage les conclusions, mis à part la thèse que la position de Platon se veut un dépassement de celle de Xénophane (il s'agit plutôt, à mes yeux, d'un retour) et, en outre, le fait qu'elle omet de mettre en valeur, dans le cas de Platon, l'aspect de la convenance du discours. 
images plus infidèles qu'une autre, il faut nous en contenter, en nous souvenant que moi qui parle et vous qui êtes mes juges sommes d'humaine nature, de sorte que, si, en ces matières, on nous propose un mythe vraisemblable, il ne sied pas de chercher plus loin ${ }^{38}$.

On voit que les vecteurs de la ressemblance qui se concentrent dans

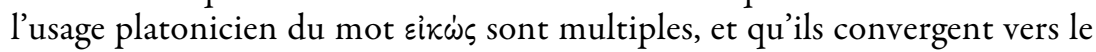
sens de convenance qui est propre au mot depuis Homère. En fait, le discours sur la physis tire sa plausibilité de la somme de plusieurs facteurs: (a) il ressemble à son objet (qui, notons-le, est un objet caché à la vue,

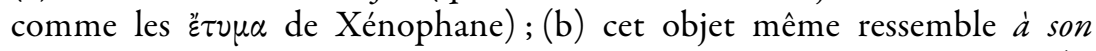
modèle, en vertu de la présupposition téléologique qui gouverne le Timée (le démiurge vise à construire un monde le plus semblable possible au vivant intelligible); (c) il est convenable ( $\pi p \varepsilon \dot{z} \pi \varepsilon l$ que ce discours, en adaptant ses prétentions épistémiques au statut ontologique inférieur de son objet aussi bien qu'à la nature humaine, n'exige pas la certitude absolue sur toutes choses, d'autant plus qu'il peut bien trouver de la satisfaction dans ses propres constructions ( $\dot{\alpha} \gamma \alpha \pi \tilde{\alpha} \nu \chi \chi p \dot{\eta}$ indique, me semble-t-il, un sentiment plus positif que la résignation). On pourrait enfin décrire cette situation épistémologique en disant que Platon y enrichit la dichotomie parménidéenne entre le sensible et l'intelligible par une théorie élaborée de la relation entre les deux niveaux, et qu'il remet en valeur dans ce nouveau cadre la réflexion de Xénophane sur le statut du discours physique, qui, lui aussi, voulait être à la fois « raisonnable » et « approprié » à son champ particulier ${ }^{39}$.

Je voudrais conclure sur un autre indice fort significatif d'une influence de Xénophane qu'on peut retrouver dans le Timée. Rappelons que Xénophane avait modelé sa construction de la figure du dieu sur un critère de theoprepeia, voire de convenance aussi bien rationnelle que morale. Or, ce même critère est appliqué dans le Timée (30a6-b8) à la construction du monde (qui est, rappelons-le, lui aussi un dieu) par le démiurge. On lit ici, en effet, qu'il n'est pas «permis » de penser que le démiurge ait opéré, étant donné sa bienveillance, sans viser au meilleur monde possible $\left(\theta \dot{\varepsilon} \mu \iota \varsigma \delta^{\prime}\right.$

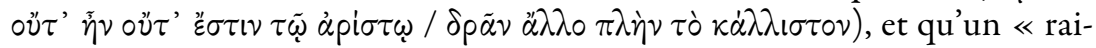
sonnement plausible $\gg(\kappa \alpha \tau \dot{\alpha} \lambda$ óyov $\tau$ òv $\varepsilon$ ikò $\tau \alpha)$, voire fondé sur la considération de l'action qu'il est le plus approprié d'attribuer au démiurge en vue de l'ordre et de la beauté de l'univers, assure qu'il a donné au monde une âme et une intelligence suivant un projet bienveillant. Pour la même

38. Les traductions du Timée sont celles de Brisson 1992.

39. Les deux sens coexistent et se renforcent mutuellement; voir p. ex. la lecture de Burnyeat 2005. Burnyeat rappelle en passant, p. 148 n. 13 et p. 153, que Parménide B8.6061 manifeste la préoccupation semblable d'un discours avant tout «approprié ». Betegh 2010 et Mourelatos 2010 partent tous les deux de l'interprétation de Burnyeat. 
raison, on lit en $33 \mathrm{~b} 1$ qu'il a également modelé le corps du monde selon cette forme la plus convenable qu'est la sphère, sa nature étant de se suffire, parfaitement accompli en soi-même. Tout le passage qui suit est un tissu de variations sur ce motif, visant à justifier la forme sphérique de l'univers.

Plat. Tim. 33b1-d5

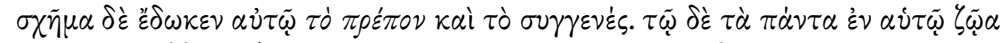

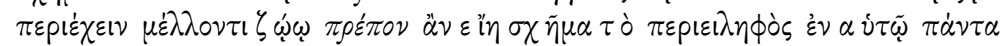

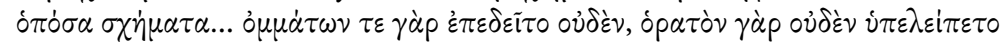

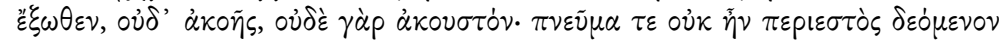

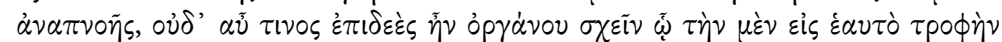

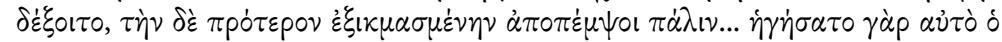

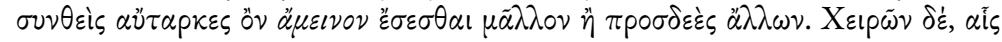

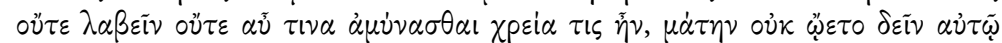

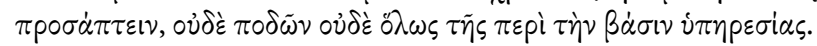

Comme figure, il lui donna celle qui lui convenait et qui lui était apparentée. Au vivant qui doit envelopper en lui-même tous les vivants, la figure qui pourrait convenir, c'était celle où s'inscrivent toutes les autres figures. Aussi est-ce la figure d'une sphère, dont le centre est équidistant de tous les points de la périphérie, une figure circulaire ... le monde n'avait nullement besoin d'yeux, car il ne restait rien à voir à l'extérieur de lui ; ni d'oreilles, car il y avait non plus rien à entendre à l'extérieur de lui. Et nul souffle ne l'entourait, qui attendît qu'on le respire. Par ailleurs, il n'avait pas besoin d'un organe, qui lui permette d'absorber de la nourriture, puis de rejeter cette nourriture après en avoir extrait les sucs ... En effet, celui qui l'a constitué a considéré que le monde serait bien meilleur s'il se suffisait à luimême plutôt que s'il était dépendant de quoi que ce soit. Le dieu décida que, comme ces instruments ne présenteraient aucune utilité, il devait se garder de lui adapter des mains, qui lui permissent de saisir ou de repousser quelque chose, des pieds ou plus généralement tout appareil servant à se tenir debout.

Ajoutons que le monde n'a pas besoin de pieds parce que le dieu lui a assigné, parmi les mouvements d'un corps physique, celui qui lui est appro-

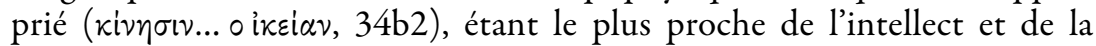
pensée, à savoir un mouvement de rotation uniforme dans le même lieu. En somme, bien qu'étant un être vivant et pensant, ce monde n'a pas besoin des fonctions et des organes dont ont besoin les animaux et les hommes pour vivre, sentir et agir. Cornford n'a pas manqué de reconnaître dans cette description, dans une page souvent négligée de son commentaire ${ }^{40}$, un écho de Parménide (fr. B8.26-33, où l'immutabilité de l'Être est liée également à son autonomie absolue), du Sphairos d'Empédocle (fr. B29), et de Xénophane. En effet, celui-ci insiste dans nombre de fragments sur le

40. À la brillante exception de Palmer 1999, p. 193-197. Cf. Cornford 1937, p. 54-57. 
fait qu'il ne faut au dieu ni organes spécifiques poussant de sa forme sphé-

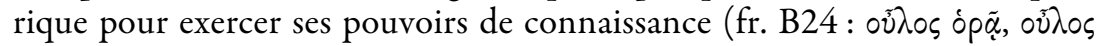

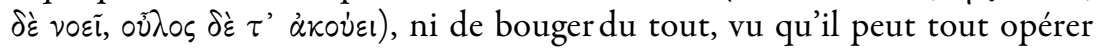

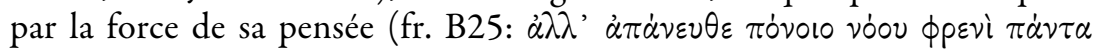

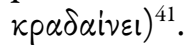

L'impression que l'influence de Xénophane est prépondérante dans ce passage du Timée peut servir d'appui à mon hypothèse plus générale, selon laquelle c'est de Xénophane que Platon tire les critères fondateurs de sa construction cosmologique. Dans ce contexte particulier il ne ferait qu'appliquer au monde, qui est divin, la catégorie de la convenance du discours que Xénophane avait élaborée en rapport avec la définition de la nature du dieu. Pour conclure, bien que l'influence du savoir présocratique sur le dialogue cosmologique n'ait pas encore été explorée en détail, il est sûr que Platon y puise largement, dans le programme d'une enquête qui se veut tout autre chose qu'une parodie. L'interprétation proposée des divers usages d'eixw's dans les trois contextes que nous avons examinés pourrait aboutir à la conclusion que, pour le Platon qui se consacre dans ses dernières années à l'étude de la nature, Xénophane l'emporte sur Parménide ${ }^{42}$.

41. Le fragment 23, mentionné ci-dessus, p. 21, est également très important dans ce contexte.

42. Je ne veux évidemment pas sous-estimer l'importance de la réception de Parménide jusqu'aux dialogues les plus tardifs de Platon. Voir sur ce point en général Palmer 1999, et en particulier ici, p. 25-27, une évaluation attentive du rapport Xénophane-Parménide-Platon du point de vue du rôle du dókos. 


\section{BIBLIOGRAPHIE}

Année, M. 2012 : Parménide, Fragments, Poème précédé de Énoncer le verbe être, Paris, 2012 (Bibliothèque des textes philosophiques).

Babut, D. 1974 : «Sur la "théologie" de Xénophane », Revue Philosophique, 164 (1974), p. 401-440 (= Parerga. Choix d'articles de Daniel Babut (1974-1994), Lyon, 1994 [Collection de la Maison de l'Orient méditerranéen, Série littéraire et philosophique], p. 47-86).

Betegh, 2010: «What Makes a Myth eikôs? Remarks Inspired by Myles Burnyeat's EIKOS MYTHOS », dans Mohr-Sattler 2010, p. 213-224.

BOEKE, H. 2007 : The Value of Victory in Pindar's Odes. Gnomai, Cosmology and the Role of the Poet, Leyde, 2007 (Mnemosyne. Supplementum, 285).

Brisson, L. 1992 : Platon, Timée/Critias. Traduction inédite (avec la collaboration de M. Patillon), introduction et notes, Paris, 1992 (GF 618).

- 2012: «Why is the Timaeus called an eikôs mythos and an eikôs logos ? », dans Collobert-Destrée-Gonzalez (éd.) 2012, p. 369-391.

Bryan, J. 2012 : Likeness and Likelihood in the Presocratics and Plato, Cambridge, 2012 (Cambridge Classical Studies).

BURNYEAT, M. 2005 : «EIK $\Omega \Sigma$ MY®O $\Sigma$, Rhizai : A Journal for Ancient Philosophy and Science, 2, 2005, p. 143-165 (= C. Partenie (éd.), Plato's Myths, Cambridge, 2009, p. 167-186).

Chantraine, P. 1990 : Dictionnaire étymologique de la langue grecque, Paris, 1990.

COLE, Th. 1983: « Archaic Truth », Quaderni Urbinati di Cultura Classica, 42, N. S. 13 (1983), p. 7-28

Collobert, C., P. Destrée, F. J. Gonzalez (éd.) 2012 : Plato and Myth, Studies on the Use and Status of Platonic Myths, Leyde, 2012 (Mnemosyne. Supplementum, 337).

CORCElla, A. 1984 : Erodoto e l'analogia, Palerme, 1984.

Cordero, N.-L. 2010 : « The 'Doxa of Parmenides' Dismantled », Ancient Philosophy, 30 (2010), p. 231-246.

CORnFORD, F. M. 1937 : Plato's Cosmology. The Timaeus of Plato translated with a running commentary, Londres, 1937, 19564.

CURD, P. 1998 : The Legacy of Parmenides. Eleatic Monism and Later Presocratic Thought, Princeton 1998, Las Vegas 20042.

FRÄNKEL, H. 1921 : Die homerische Gleichnisse, Goettingue, 1921.

- 1925: «Xenophanesstudien, II : Die Erkenntniskritik des Xenophanes », Hermes, 60 (1925), p. 180-192 (= H. Fränkel, Wege und Formen frühgriechischen Denkens. Literarische und philosophiegeschichtliche Studien, Munich, 1955 [19602, 19683], p. 335-349).

FrANCO RePELLINI, F. 2003 : « La linea e la caverna », dans M. Vegetti (éd.), Platone, $L a$ Repubblica, Traduzione e commento, vol. V, Rome, 2003 (Elenchos, 28-5), p. 355403.

Gerber, D. E. 1982 : Pindar's Olympian One : A Commentary, Toronto/Buffalo/Londres, 1982 (Phoenix. Supplementary volume).

GersON, L. 2009 : Ancient epistemology, Cambridge, 2009 (Key themes in ancient philosophy, 1).

Grasso, E. 2012 : « Myth, Image and Likeness in Plato's Timaeus », dans Collobert-Destrée-Gonzalez (éd.), 2012, p. 343-367.

Hoffman, D. C. 2008 : «Concerning Eikos: Social Expectation and Verisimilitude in Early Attic Rhetoric », Rhetorica, 26 (2008), p. 1-29.

Hussey, E. 1990: «The Beginnings of Epistemology», dans S. Everson (éd.), Epistemology, Cambridge, 1990 (Companions to Ancient Thought, 1), p. 11-38. 
- 2006: « The Beginnings of Science and Philosophy in Archaic Greece », dans M. L. Gill \& P. Pellegrin (éd.), A Companion to Ancient Philosophy, Malden, MA, 2006 (Blackwell Companions to Philosophy), p. 3-19.

JAEGER, W. 1953 : Die Theologie der frühen griechischen Denker, Stuttgart, 1953. [Trad. fr. À la naissance de la théologie, essai sur les présocratiques, Paris, 1966 (Cogitatio fidei, 19).]

LESHER, J. H. 1992 : Xenophanes of Colophon, Fragments. A Text and Translation with a Commentary, Toronto/Buffalo/Londres, 1992 (Phoenix. Supplementary Volume : The Phoenix Presocratics, 4).

LLOYD, G. E. R. 1966 : Polarity and Analogy: Two Types of Argumentation in Early Greek Thought, Cambridge, 1966.

- 1996: Adversaries and authorities. Investigations into ancient Greek and Chinese science, Cambridge, 1996.

LloYd, G.-Sivin, N. 2002: The Way and the Word. Science and Medicine in Early China and Greece, Londres, 2002.

Mansfeld, J. \& Primavesi, O. 2011 : Die Vorsokratiker. Griechisch/Deutsch. Ausgewählt, übersetzt und erläutert, Stuttgart, 2011 (Reclam-Bibliothek).

MoHr, R. D. \& Sattler, B. M. (éd.) 2010 : One book, the whole universe: Plato's Timaeus today, Las Vegas/Zürich/Athènes, 2010.

Mourelatos, A. P. D. 2008 : The Route of Parmenides: A Study of Word, Image, and Argument in the Fragments (11970), revised and expanded edition, Las Vegas/Zürich/Athènes, 2008.

- 2010 : «The Epistemological Section (29b-d) of the Proem in Timaeus' Speech: M. F. Burnyeat on eikôs mythos, and comparison with Xenophanes B 34 and B $35 »$, dans Mohr \& Sattler 2010, p. 225-247.

Palmer, J. A. 1999 : Plato's reception of Parmenides, Oxford, 1999.

PRIMAVESI, O. 2005: «Theologische Allegorie: Zur philosophischen Funktion einer poetischen Form bei Parmenides und Empedokles », dans M. Horster \& C. Reiz (éd.), Wissensvermittlung in dichterischer Gestalt, Stuttgart, 2005 (Palingenesia, 85), p. 69-93.

RIVIER, A. 1952: Un emploi archaïque de l'analogie chez Héraclite et Thucydide, Lausanne, 1952 (Collection des Études de Lettres. 11).

- 1956: «Remarques sur les fragments 34 et 35 de Xénophane », Revue de philologie, de littérature et d'histoire ancienne, 30 (1956), p. 37-61 (= Id., Études de littérature grecque : Théâtre, poésie lyrique, philosophie, médecine [F. Lasserre \& J. Sulliger éd.], Genève, 1975 [Publications de la Faculté des Lettres de Lausanne, 21], p. 337 367).

SASSI, M. M. 2005 : « Da Senofane al Timeo : il problema del discorso "verosimile" », dans M. Bugno (éd.), Senofane ed Elea tra Ionia e Magna Grecia, Naples, p. 141-146.

- 2011a : «Senofane fra i Sofisti. Dai limiti della conoscenza (21 B 34 DK) al paradosso eristico (Plat., Men., 80d5-e5 », Methexis, 24 (2011), p. 7-20.

-2011b: Gli inizi della filosofia: in Grecia, Turin, 2011 (Nuova Cultura).

— (à paraître) : «Where epistemology and religion meet. What do(es) the god(s) look like? », à paraître dans Rhizomata : A Journal for Ancient Philosophy and Science, 2 (2013).

SCHIESARO, A. 1990 : Simulacrum et imago. Gli argomenti analogici nel De rerum natura, Pise, 1990 (Biblioteca di materiali e discussioni per l'analisi dei testi classici, 8).

SNELL, B. 1946: Die Entdeckung des Geistes. Studien zur Entstehung des europäischen Denkens bei den Griechen, Hambourg, 1946.

TURrini, G. 1977 : «Contributo all'analisi del termine eikos. I. L'età arcaica », Acme, 30 (1977), p. 541-558. 
- 1979: «Contributo all'analisi del termine eikos. II. Linguaggio, verosimiglianza e immagine in Platone », Acme, 32 (1979), p. 299-323.

VERDENIUS, W. J. 1942 : Parmenides: some comments on his poem, Groningen, 1942.

- 1988: Commentaries on Pindar, vol. II, Leyde/New York, 1988 (Mnemosyne. Supplementum, 101). 